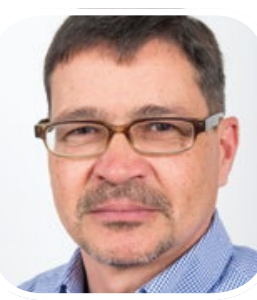

By Mark

\section{Foster of}

the Child

Protection

Company

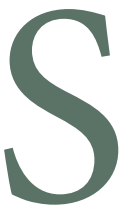

ocial distancing has become the new normal for many of us in our efforts to slow the spread of COVID-19 (Coronavirus) and protect our national healthcare service during the global crisis.

Adjusting to such an unfamiliar routine and, for many, being isolated from colleagues, friends and family at a time like this can be overwhelming, especially when it comes to essential matters such as safeguarding. However, it's important to remember that you are not alone, and if you do find yourself worried about a child or vulnerable adult, there are steps you can take to reach out and safely share your concerns.

\section{Adapt to change in your dental} team's safeguarding process

All dental staff should be trained to notice the signs of abuse and neglect in children and vulnerable adults as part of their regular dental safeguarding training. Unfortunately, many of these signs and indicators are physical and are therefore much more difficult to pick up on when we are practising social distancing, with routine appointments being cancelled and emergency dental staff triaging over the phone instead.

For example, evidence of physical abuse such as bruising and burn marks might be visible during direct contact with a child any other time, but such injuries would be virtually invisible when consulting with the child's parents or carers over the phone during the current global crisis.

This does not mean your responsibility to safeguard children and vulnerable adults will be put on hold until life returns to normal. Now more than ever, dental staff need to be vigilant at spotting the signs of abuse. More time at home for individuals who suffer from abuse means more opportunity for things to go wrong. Children and adults at risk are in an incredibly vulnerable position during social isolation, and it is your duty as a dental professional to adapt your safeguarding processes with this in mind.

\section{Know who your Safeguarding Lead} is and how to contact them

Every dental team should have a designated member of staff who takes a lead responsibility for safeguarding. In larger practices, there may be more than one lead person in this role.

At all times, the name and contact details of your practice's Safeguarding Lead should be displayed in a prominent location throughout staff areas (such as in the toilets, staff rooms, and inside surgery rooms). These contact details should also be listed in your practice's safeguarding folder for all members of staff to access at any time.

If your team is currently working from home because the dental practice has been closed temporarily, it is incredibly important that you make arrangements so that these contact details are still easily accessible to everyone on your team. We suggest creating an online safeguarding folder accessible to all staff in a private online location on your system. Ensure that everyone knows how to $\log$ in and access the information remotely, so that if a concern arises while they are working from home, they have the means to act.

If your practice already has an online safeguarding folder, you will need to confirm that all contact details are up to date. Also be sure to check that your local authority and partner services are still using the same contact details during lockdown, as your safeguarding folder will need to include any temporary contact information or new processes in case there is a need for referral. 
Respond to sick and/or furloughed staff with safeguarding in mind If your Safeguarding Lead is furloughed, or if they have to take time out of work during lockdown and will not be contactable, you should make arrangements within your team to ensure another member of staff is prepared to take over the role.

We offer accredited Level 1 and Level 2 online safeguarding courses at the Child Protection Company, developed by experts specifically for dental teams and recommended by the British Dental Association (BDA). These online courses take around one to two hours to complete in full, with a verifiable certificate available to download immediately upon successful completion.

If you need to train staff in a hurry, do so with the confidence that you're not compromising on quality. Safeguarding is an essential topic and something you will be inspected on by CQC. Fortunately, our certificates are valid for two years, so you will be able to return to work after lockdown with the confidence that your safeguarding training is up to date and accredited.

Each of these online dental courses is also worth three hours of verifiable CPD under the General Dental Council Lifelong Learning Scheme.

You can visit us at www.

childprotectioncompany.com to view our online safeguarding training options or start a course immediately.

\section{Be on high alert for safeguarding concerns}

Even though you are not likely to be seeing your patients in person during this time, there are still many ways that you can keep an eye out for safeguarding concerns. It is especially important to be vigilant with this right now - after all, you could be the only trained professional a child or at-risk adult has contact with for months. Where school staff or friends might have noticed signs of abuse and neglect before, such individuals could now be completely vulnerable to maltreatment.

A dental safeguarding training course will introduce you to the various signs and indicators of abuse and neglect, including the behavioural changes that might suggest something is wrong. We recommend you familiarise yourself with these signs and keep them in mind during contact with all patients.

Remember: Anyone can be a victim of abuse, at any age, at any time.

Some of the non-physical signs you should pay attention to - though this is not an exhaustive list by any means - include:

- Family members or carers who insist on speaking for your patient over the phone, especially those who get agitated or angry when you ask to speak directly to the patient, or those who refuse to hand the call over

- Any obvious signs of distress in the background of phone calls (eg shouting, screaming, crying, general disturbance)

- Inconsistent or concerning answers to general questions

- A change in tone or behaviour when it is obvious there is somebody else in the room, or when the other person leaves the room

- Jokes about abusive behaviour or neglect (eg 'I could kill them...')

- Missed calls or no contact, especially on multiple occasions or when a patient is aware of their scheduled appointment

- Any disclosure of mistreatment, no matter how small (remember, this could be the only 'safe' information a child or adult at risk feels comfortable to tell you, while
If you feel that an individual is at immediate risk of danger or harm, you must treat it as an emergency and dial 999 in the first instance.

\section{Remember that you are not alone} Safeguarding is everybody's business, and even during times of social distancing, you are never alone. Have the confidence to report any concerns and ensure that everyone on your team is aware of how to do so. There is no such thing as a 'wrong' referral when it comes to safeguarding - even if no evidence of abuse or maltreatment is found, you will at least have the peace of mind that everyone is safe.

However, if your referral leads to an investigation, you could quite literally be responsible for saving somebody's life.

Needless to say, being mindful of what to do in the event of a safeguarding incident and knowing ahead of time who to reach out to with your concerns is paramount. Effective safeguarding is a group effort, and now more than ever, we all need to do our best to ensure that everyone is kept safe.

\section{'Safeguarding is everybody's business,} and even during times of social distancing, you are never alone. Have the confidence to report any concerns and ensure that everyone on your team is anvare of how to do so.'

any number of bigger problems could be ongoing alongside this smaller incident)

- Family members or carers who try to explain any maltreatment or injury the child or vulnerable adult has mentioned to you with inconsistent or unbelievable explanations

- Deflecting questions or refusing to talk.

Every concern you have, no matter how small or seemingly insignificant, should be recorded along with the date and time, a brief description of what happened, and any other relevant information. This information should be kept confidential as per your practice's safeguarding process, and you should get in touch with your Safeguarding Lead for advice on what to do next.

\section{Reach out to us to arrange remote safeguarding training}

Our team at the Child Protection Company is currently working from home, but our friendly customer support team is available from 9 am to $5.30 \mathrm{pm}$, Monday to Friday, to answer your questions and advise dental teams on the best choice for verifiable, socially distanced safeguarding training.

Please get in touch with us by calling 01327552030 or email us at help@ childprotectioncompany.com.

Alternatively, you can visit our website at www.childprotectioncompany.com to browse our accredited courses and start safeguarding training today.

https://doi.org/10.1038/s41407-020-0315-z 